Results Estimates of exposure for 138 EMF sources were obtained from measurements (1424 aggregated records) extracted from 71 papers and hygiene reports (1974-2013). For each source, exposure was calculated by frequency band and dosimetry type, as the arithmetic and geometric means of all measurements identified. Standard deviations were included in order to characterise the variability of the estimates.

Conclusions A source-exposure matrix has been constructed for the most common sources of EMF in the workplace, based on the responses to the INTERPHONE-INTEROCC study questionnaire. This database currently represents the most comprehensive source of information on occupational EMF exposure and is available on request to researchers.

\section{OCCUPATIONAL USE OF INSECTICIDES, FUNGICIDES AND FUMIGANTS AND RISK OF NON-HODGKIN LYMPHOMA AND MULTIPLE MYELOMA IN THE AGRICULTURAL HEALTH STUDY} ${ }^{4} J o s e p h$ Barker, ${ }^{4}$ Dennis Buckman, ${ }^{5}$ Kent Thomas, ${ }^{6}$ Dale Sandler, ${ }^{7} J a n e$ Hoppin, ${ }^{1}$ Stella Koutros, 'Gabriella Andreotti, 'Jay Lubin, 'Aaron Blair, ${ }^{1}$ Laura Beane Freeman. ${ }^{1}$ Occupational and Environmental Epidemiology Branch, National Cancer Institute, Bethesda, MD, USA; ${ }^{2}$ University of lowa, Department of Epidemiology, lowa City, IA, USA; ${ }^{3}$ National Institute for Occupational Safety and Health, Cincinnati, OH, USA; ${ }^{4} I M S$, Calverton, MD, USA; ${ }^{5}$ National Exposure Research Laboratory, U. S. Environmental Protection Agency, Research Triangle, NC, USA; ${ }^{6}$ National Institute of Environmental Health Sciences, Research Triangle, NC, USA; ${ }^{7}$ North Carolina State University, Raleigh, NC, USA

\subsection{6/oemed-2014-102362.111}

Objectives Farming and exposure to pesticides have been linked to non-Hodgkin lymphoma (NHL), and multiple myeloma $(\mathrm{MM})$ in previous studies. We evaluated use of insecticides, fungicides and fumigants and risk of NHL, including MM and other NHL sub-types in the Agricultural Health Study, a US-based prospective cohort study.

Method A total of 527 cases occurred among 55875 pesticide applicators from enrollment (1993-1997) through 2011 in Iowa and 2010 in North Carolina. Information on pesticide use, other agricultural exposures and other factors was obtained from questionnaires at enrollment and follow-up approximately five years later (1999-2005). Information from these questionnaires was used to create lifetime-days and intensity-weighted lifetime-days of pesticide use. Poisson regression and polytomous logit models were used to calculate relative risks (RR) and 95\% confidence intervals (CI) to evaluate associations between 26 pesticides and NHL and five NHL-subtypes including multiple myeloma, while adjusting for potential confounding factors.

Results Statistically significant positive exposure-response trends occurred between overall NHL risk and lindane ( $\mathrm{p}$-trend = 0.004 ) and DDT ( $\mathrm{p}$-trend $=0.02$ ). In addition, ever use of terbufos was associated with NHL overall $(\mathrm{RR}=1.2 ; \mathrm{CI}=1.0-1.5)$, but with no exposure-response trend. In sub-type analyses, terbufos and DDT were associated with small cell lymphoma/ chronic lymphocytic leukaemia/marginal cell lymphoma. In addition, lindane and diazinon were associated with follicular lymphoma and permethrin with MM although tests of homogeneity did not show significant differences in exposure-response among NHL-subtypes for any chemical.

Conclusions These findings are among the first to suggest links between DDT, lindane, permethrin, diazinon and terbufos and specific NHL subtypes.

\section{GENE-SPECIFIC DNA METHYLATION AS A VALUABLE TOOL FOR RISK ASSESSMENT: THE CASE OF OCCUPATIONAL EXPOSURE TO DIFFERENT VOC'S IN MEXICAN WORKERS}

${ }^{1}$ Octavio Jiménez-Garza, ${ }^{2}$ Andrea Baccarelli, ${ }^{2}$ Hyang-Min Byun, ${ }^{3}$ Giovanni Battista Bartolucci, ${ }^{3}$ Mariella Carrieri. 'University of Gunajuato Campus León, Health Sciences Division, Léon, Guanajuato, Mexico; ${ }^{2}$ Harvard School of Public Health, Laboratory of Human Environmental Epigenomics, Boston, Massachusetts, USA; ${ }^{3}$ University of Padova, Department of Medicine, Padova, Italy

\subsection{6/oemed-2014-102362.112}

Objectives To determine gene-specific methylation levels (promoter region) on genes from critical cellular pathways in persons occupationally exposed to a single volatile organic compound (VOC) or to a mixture of them

Method Workers from two tanneries, two shoe factories, and two gas stations were included (exposed groups to VOC's). We also included administrative workers (reference group), all of them from the city of León Guanajuato, México. In tannery workers we measured individual exposure levels to three different VOC's; for the rest of the groups we measured up to 7 VOC's. After exposure characterisation, we took blood samples and extracted DNA in order to determine, by PCR-pyrosequencing, methylation levels in genes involved in inflammation, DNA repair, oxidative stress and xenobiotic metabolism pathways.

Results Only toluene environmental levels were higher in tannery workers. Workers from the leather shoe factory showed the highest exposure levels for up to five different VOC's. There was no statistical significant difference in gene-specific methylation for tannery workers when compared to the control group. For the leather shoe factory workers, we found hypermethylation in the TNFa, SOD1 and TOP2A promoter regions compared to the control group or to other exposed groups. Gas station attendants showed hypermethylation for the IL6 gene compared to controls Conclusions Occupational exposure to a mixture of VOC's has important effects on the methylation status of genes involved in inflammation, DNA repair and oxidative stress. These epigenetic changes, detectable at a pre-clinical stage, represent a valuable tool for performing an early risk assessment in these populations

\section{PREVENTING NEEDLESTICKS AND OTHER SHARPS INJURIES TO HOME CARE AIDES: RESULTS OF A SURVEY TO IDENTIFY HAZARDS DURING HOME VISITS}

${ }^{1}$ David Kriebel, ${ }^{1}$ Natalie Brouillette, 'Pia Markkanen, 'Catherine Galligan, 'Susan Sama, ${ }^{1}$ Rebecca Gore, ${ }^{2}$ Angela Laramie, 'Daniel Okyere, ${ }^{1}$ Chuan Sun, ${ }^{2}$ Letitia Davis, ${ }^{1}$ Margaret Quinn. 'University of Massachusetts Lowell, Lowell, MA, USA; ${ }^{2}$ Massachusetts Department of Public Health, Boston MA, USA

\subsection{6/oemed-2014-102362.113}

Objectives To follow up on our previous finding that home care (HC) aides have a substantial risk of injury with used sharp medical devices, this study's goal was to identify modifiable aspects of aide-client encounters during HC visits that increase risk of sharps injuries (SI).

Method A survey of 1249 HC aides was conducted in eastern Massachusetts. Approximately half the participants (634) were employed by HC agencies, and half (615) directly by HC clients and their families. A questionnaire gathered data on aides' most recent home visits, enabling quantification of hazardous working conditions. The specific investigation described here focused on understanding determinants of hazardous conditions likely to increase the risk of SI including: the aide finding used sharps 